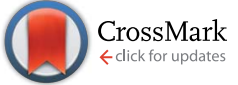

Cite this: Chem. Sci., 2016, 7, 3137

\title{
Proton-triggered switch based on a molecular transistor with edge-on gate $\dagger$
}

\author{
Lianwei Li,\$ Wai-Yip Lo,\$ Zhengxu Cai, Na Zhang and Luping Yu*
}

The manipulation of charge transport through single molecules so that electronic information can be controlled is a basic challenge that is important for both fundamental understanding of the mechanisms and the potential applications in single-molecule technologies. This paper reports the influence of protonation on the gating effect in a series of molecular wires utilizing a pyridinoparacyclophane (PPC) moiety as the edge-on gate. It was found that the molecular conductance, transition voltage, and the corresponding tunnelling barriers can be reversibly switched by the protonation/deprotonation process of the nitrogen atom on the PPC pyridine ring. It was found that protonation levels off the tunnelling barrier of different molecules and converts $p$-type molecular wires into n-type, reversibly.

Received 13th January 2016

Accepted 19th January 2016

DOI: $10.1039 /$ c6sc00152a

www.rsc.org/chemicalscience

group changes from a strong electron acceptor to a strong

\section{Introduction}

It is now known that the variation of molecular structures, either electronic or geometric changes, can exert a profound effect on the electrical conductance of single molecular wires. For example, a meta- or para-position of connection will lead to different electrical conductance; and the introduction of a dipole into single molecules can alter the molecules rectification property.$^{1-3}$ In addition to these intrinsic structural changes, there is a continuing interest in molecular electronics to build devices which integrate molecules whose conductivity can be manipulated by external stimuli such as the $\mathrm{pH}$ values of environmental solutions, ${ }^{2,4}$ light, ${ }^{5}$ mechanical motion, ${ }^{6}$ temperature, ${ }^{7}$ magnetic fields, ${ }^{8}$ or electric fields. ${ }^{9-16}$ For example, we have recently demonstrated the concept of edge-on gating at the molecular level with a set of single molecular wires, which contain a 2.2(2,6)pyridinoparacyclophane-1,9-diene (PPC) moiety (Fig. 1a). ${ }^{17}$ In this system, we took the advantage of the perpendicular orientation between the two aromatic planes and used the pyridine end of the moiety as the gating part of the wire attached with functional groups of different electronic demands. ${ }^{18,19}$ Further studies by the scanning tunneling microscopy break-junction (STM-BJ) technique demonstrated that the single-molecule conductance, the orbital energy level, and the charge tunneling barrier of these molecular wires can be tuned by changing the electronic properties of the gating substituent group. The collective behavior of these compounds resembles that of field effect transistors when the functional

Department of Chemistry and the James Franck Institute, The University of Chicago, 929 E 5 $7^{\text {th }}$ Street, Chicago, IL 60637, USA. E-mail: lupingyu@uchicago.edu

$\dagger$ Electronic supplementary information (ESI) available: XPS spectra, contact angle results, UV-Vis spectra, ${ }^{1} \mathrm{H}-\mathrm{NMR}$ spectra, STM-BJ results. See DOI: 10.1039/c6sc00152a

\$ These authors contributed equally to this work.

\section{electron donor.}

An important function of a transistor is its switching behavior, which is the basis of the modern computing mechanism. The function of switch "on" and "off" corresponds to the binary information of " 0 " and " 1 ". In this paper, we report an on/off switch based on a molecular wire containing the edge-on pyridinoparacyclophane moiety, triggered by the protonation of the pyridine ring. The results reveal that the protonation/ deprotonation processes on the nitrogen atom of the edge-on pyridine ring can reversibly alter the electrical properties of the molecular wire, leading to a binary system.

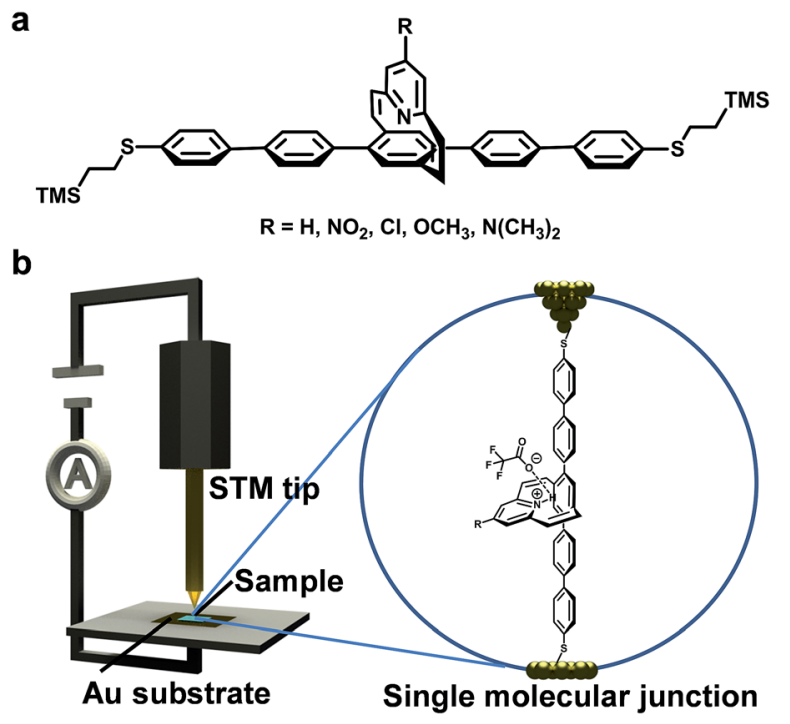

Fig. 1 Experimental setup. (a) Chemical structure of the PPC-based molecular wires. (b) A representation of the metal-molecule-metal junction in the STM-BJ technique. 


\section{Results and discussion}

The detailed synthesis and characterization of these PPCmolecular wires with a gating pyridinyl moiety whose paraposition is functionalized with groups $-\mathrm{NO}_{2},-\mathrm{Cl},-\mathrm{H},-\mathrm{OCH}_{3}$, and $-\mathrm{N}\left(\mathrm{CH}_{3}\right)_{2}$ were described previously. ${ }^{17}$ The pyridinoparacyclophane unit was shown to be similar to other pyridine derivatives that can be protonated by strong acid. ${ }^{18,20,21}$ To measure the conductance of single molecules, self-assembled monolayer (SAM) films of the deprotected thiol molecules were prepared on a gold surface via thiol-gold binding chemistry. The resulting SAM was further treated with trifluoroacetic acid (TFA) (100 mM in degassed THF) to protonate the SAM for conductance measurements, and then with $\mathrm{Na}_{2} \mathrm{CO}_{3}$ solution (100 $\mathrm{mM}$ in a degassed water/acetonitrile ( $\mathrm{v} / \mathrm{v}, 1 / 1)$ mixture) to obtain the deprotonated assembly. Details of the preparation of the SAMs can be found in the ESI. $\dagger$ The successful immobilization of these molecular wires onto gold substrates was confirmed by the CV measurements ${ }^{17}$ and the XPS characterization (ESI Fig. S1 and Table S1 $\dagger$ ), and was further supported by the variation in the water droplet contact angles of these modified surfaces compared with a bare gold surface (ESI Fig. S2 $\dagger$ ). Specifically, the XPS studies indicated that the $\mathrm{C} / \mathrm{S}$ ratio for the $\mathrm{PPC}-\mathrm{OCH}_{3}$ SAM before treatment with TFA was 40.0/2.3, in agreement with the theoretical ratio of 40.0/2.0. An extra signal of fluorine was detected for the TFA-treated PPC$\mathrm{OCH}_{3}$ SAM, demonstrating the success of the protonation process.

The single-molecule conductance was measured by the break-junction technique using a modified STM system. ${ }^{13,22,23}$ The experiments were carried out at a small bias voltage of $100 \mathrm{mV}$ in degassed toluene. ${ }^{22}$ The conductance traces of the molecular junctions with different substituents were recorded using a LabVIEW program. Fig. 2a shows the typical conductance-distance curves for the protonated single molecular junctions with different functional groups. The plateaus of constant conductance observed in these stretching traces at around $\sim 10^{-5} G_{0}$ represent the signature of a single molecule junction, where $G_{0}$ is the quantum of conductance $2 e^{2} / h$ ( $e$ is the electron charge and $h$ is the Planck constant). The plateau length is generally around $0.5-1.0 \mathrm{~nm}$. The fluctuation of the conductance value below $10^{-6} G_{0}$ originates from the noise floor. Repeated measurements gave a statistical assessment of the junction properties. Fig. $2 \mathrm{~b}$ shows the corresponding conductance histograms generated from 300-500 effective conductance decay curves. The histogram reveals an unambiguous peak for all of the molecules measured. By fitting these peaks to a Gaussian function, we can determine the most probable conductance value for the junctions. More specifically, the values of the single molecule conductance of the protonated molecules PPC- $\mathrm{NO}_{2}$, PPC-Cl, PPC-H, PPC-OCH $\mathrm{O}_{3}$, and PPC$\mathrm{N}\left(\mathrm{CH}_{3}\right)_{2}$ at $100 \mathrm{mV}$ of bias voltage were determined to be $6.3 \times$ $10^{-6} G_{0}, 5.6 \times 10^{-6} G_{0}, 6.0 \times 10^{-6} G_{0}, 5.5 \times 10^{-6} G_{0}$, and $5.4 \times$ $10^{-6} G_{0}$ respectively. These results clearly indicate that the protonated molecules exhibit similar conductance, independent of the nature of the substituent on the pyridine ring. The
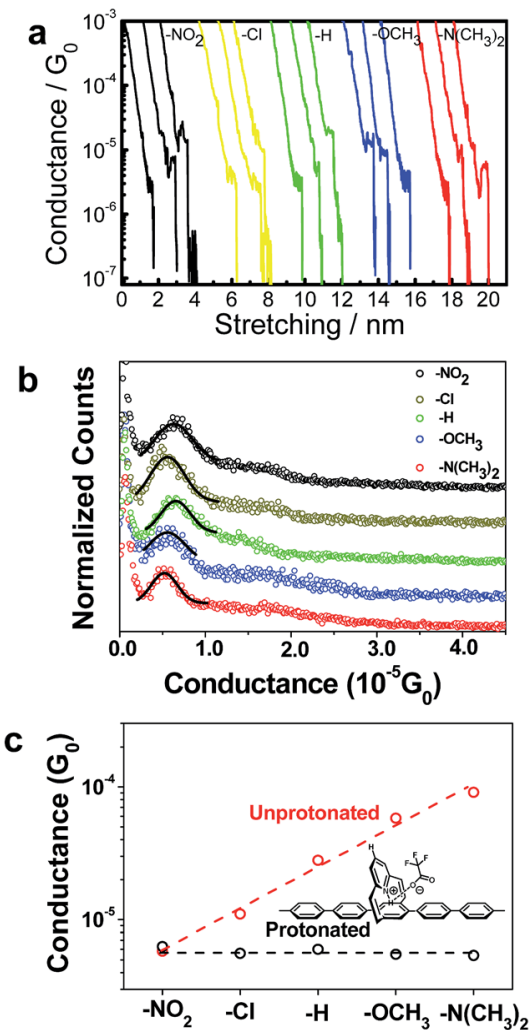

Fig. 2 Conductance of the protonated molecular junctions. (a) Representative conductance-distance traces of the protonated molecular wires with different substituents. (b) Conductance histograms of the five protonated molecular wires, where the black solid lines represent the Gaussian fitting curves. (c) The most probable conductance values of the unprotonated and protonated molecular wires. The dotted lines are only used as an eye guide.

conductance values of the protonated molecules are almost identical to that of the unprotonated, nitro-substituted compound. It shows that the positive charge on the protonated pyridinyl moiety suppressed charge transport and acted as a switch.

As mentioned in the above section, the XPS studies indicated the presence of fluorine atoms in the monolayer assembly, which is evidence for the protonation by TFA. To further ensure that the observed difference of conductance values is due to the effect of protonation, spectroscopic studies were carried out in the solution state. The ${ }^{1} \mathrm{H}$-NMR studies showed that the chemical shifts of all the protons of the PPC-based molecules were shifted to lower field after protonation, which was caused by the enhanced de-shielding effect of pyridinium compared with the neutral pyridine ring (ESI Fig. S3†). The UV-Vis spectra showed that the absorption maxima of these PPC-based molecules exhibits a bathochromic shift after TFA protonation, and that the shift enhances as the substituent group changes from an electron-withdrawing to an electron-donating group (ESI Fig. S4†). It is interesting to note that the protonation of the PPC- $\mathrm{NO}_{2}$ and PPC-Cl molecules did not lead to an obvious bathochromic shift, which is consistent with the conductance results, where all of the conductance values fell to the level of 
the nitro-substituted compound on protonation. The DFT calculations discussed below indicate that the protonation affects the LUMO more than the HOMO unless the pyridine ring is substituted with electron deficient groups.

The effect of the protonation process on the conductance of the molecular wires was shown to be reversible after the protonated SAM was treated with $\mathrm{Na}_{2} \mathrm{CO}_{3}$ solution in a water/ acetonitrile mixture. The conductance histograms of the unprotonated, protonated and deprotonated $\mathrm{PPC}-\mathrm{NO}_{2}, \mathrm{PPC}-\mathrm{H}$ and PPC-N $\left(\mathrm{CH}_{3}\right)_{2}$ molecular junctions are shown in the ESI (Fig. S5 $\dagger$ ). Fig. 3a summarizes the corresponding most probable conductance values, and the results clearly demonstrate that the deprotonation of the nitrogen atom of pyridine ring successfully restores the conductance of the neutral PPC-based molecules. The reversible features of the protonation/deprotonation process were consistent with spectroscopic observation (Fig. 3b).

The contact angle measurements showed no significant differences between the untreated SAM, and the SAMs treated by TFA and $\mathrm{Na}_{2} \mathrm{CO}_{3}$ (ESI Fig. $\mathrm{S} 6 \dagger$ ). This is reasonable because the wetting property of a SAM is mainly dominated by the outermost functional group, i.e., a thiol group in our case, instead of the pyridine ring hidden in the interior.

The protonation effect is further demonstrated by the inability of weak acid to change the conductance. The pyridine moiety cannot be protonated by acetic acid, a weak acid, with a $\mathrm{p} K_{\mathrm{a}}$ value of $\sim 4.75$. Thus, when the SAMs were treated with acetic acid, no difference in conductance was detected in the break-junction measurements (ESI Fig. S7†) before and after

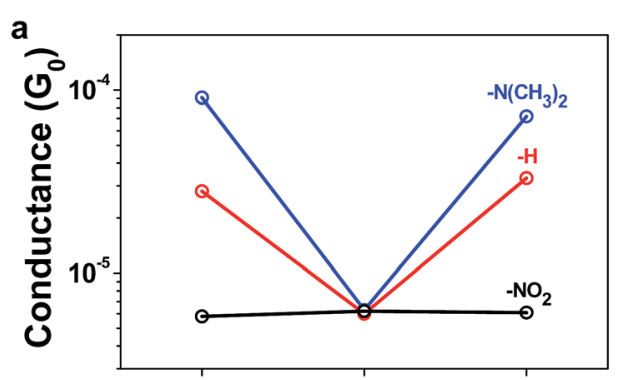

Unprotonated Protonated Deprotonated

b

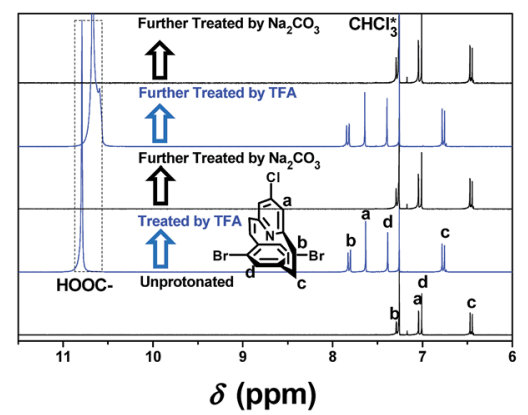

Fig. 3 Reversible change in conductance and chemical shift due to protonation. (a) The corresponding most probable conductance values of the unprotonated, protonated and deprotonated molecular wires. (b) ${ }^{1} \mathrm{H}-\mathrm{NMR}$ spectra of the PPC-Cl core of the protonation/ deprotonation cycles. protonation. A similar phenomenon was observed in all other experiments including the ${ }^{1} \mathrm{H}-\mathrm{NMR}$ spectra and UV-Vis spectra (ESI Fig. S3 and S4†).

The current-voltage $(I-V)$, conductance-voltage $(G-V)$ characteristics, and the related transition voltage spectroscopy (TVS) were studied to further probe the charge transport mechanism in the protonated molecular junctions. Details of the measurements can be found in the ESI. $\dagger$ Fig. 4 a and e plot $c a$. $800 \mathrm{I}-\mathrm{V}$ curves for the unprotonated and protonated $\mathrm{PPC}-\mathrm{H}$ molecular junctions. The incomplete $I-V$ curves constituting $\sim 70 \%$ of all the curves, due to the breakdown and instability of the molecular junctions, were automatically detected and not included in the $I-V$ histogram shown in Fig. 4. Typically, most of the initial slopes of the $I-V$ curves for the protonated $\mathrm{PPC}-\mathrm{H}$ molecular junction are smaller than that of the unprotonated version, consistent with the observed trend of the previous conductance histograms. The $I-V$ and $G-V$ histograms for the other molecules are summarized in the ESI (Fig. S8 $\dagger$ ). Most of the $I-V$ curves display a clear nonlinearity as a function of bias voltage, which is also reflected in the corresponding bowl-shaped 2-D $G-V$ histograms (Fig. $4 \mathrm{~b}$ and f). The single-molecule TVS was obtained by converting each $I-V$ curve into a curve of $\ln \left(I / V^{2}\right)$ as a function of $1 / \mathrm{V}$, the so-called Fowler-Nordheim (FN) plot. $^{23-26}$ The minimum of the FN plot corresponds to the transition voltage $V_{\mathrm{t}}$ and the 1D TVS histograms of the occurrence of the transition voltage can be constructed from these minimum values. The TVS plot of octanedithiol was constructed as a control experiment. The result indicates that the measured $V_{\mathrm{t}}$ for octanedithiol is $\sim 1.35 \mathrm{~V}$, which is consistent with the

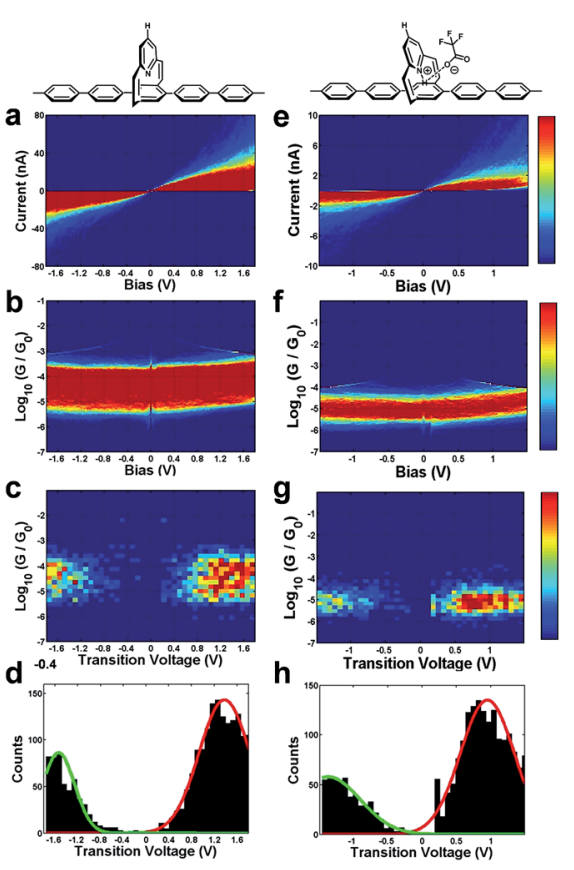

Fig. 4 Change in the electrical properties due to protonation. 2-D I-V histograms, 2-D G-V histograms, 2-D transition voltage histograms, and 1-D transition voltage histograms for the unprotonated (left column: $(a-d))^{17}$ and protonated (right column: (e-h)) PPC-H molecular junctions, respectively. 
reported values (1.30-1.40 V) in the literatures. ${ }^{23,27}$ As shown in Fig. $4 \mathrm{~d}$ and $\mathrm{h}$, the transition voltage histograms of the unprotonated and protonated $\mathrm{PPC}-\mathrm{H}$ molecules exhibit two distinguishing characteristics: (1) both histograms are asymmetric in nature; (2) the absolute values of the measured positive and negative transition voltages $\left(V_{\mathrm{t}^{+}}\right.$and $\left.V_{\mathrm{t}-}\right)$ decrease from $1.35 \mathrm{~V}$ to $0.95 \mathrm{~V}$ and from $1.51 \mathrm{~V}$ to $1.35 \mathrm{~V}$, respectively, after protonation. The asymmetry of transition voltage histograms for molecular junctions was previously observed in the work of Kushmerick ${ }^{24}$ and Tao. ${ }^{23}$ The phenomenon was mainly attributed to the metal-molecule contacts and the symmetry of the molecule itself, namely, the flat substrate electrode versus the sharp tip electrode and the stronger coupling of the $\pi$-electrons of the aromatic rings to the flat substrate than to the atomically sharp tip. Most recently, it was shown that the polarity of the solvent can also cause the asymmetry. ${ }^{28}$

Since the applied bias $V$ can affect the energy offset between the Fermi level of electrodes and molecular orbitals, $\varphi_{0} \equiv$ $\left.\varphi_{0}(V)\right|_{V=0} \rightarrow \varphi_{0}(V)$, the voltage division factor $\gamma$ was designed to account for this effect, where $\varphi_{0}(V)=\varphi_{0}+\gamma \mathrm{eV}^{.9,30}$ More specifically, the experimentally measured transition voltages $V_{\mathrm{t} \pm}$ for both bias polarities are related to the barrier height $\varphi_{0}$ (ref. 31-34) and the voltage division factor $\gamma$ based on the equations developed by Bâldea. ${ }^{29,30}$ The calculated values for the unprotonated and the protonated PPC molecular wires are summarized in Fig. 5 and ESI Table S2. $\dagger$ As shown in Fig. 5a, all the calculated $\gamma$ values are positive, indicating that the potential drop at the soft contact (e.g., the STM-tip) is slightly larger. Moreover, the $\gamma$ values of the protonated molecular wires are larger than those of the unprotonated molecular wires. This implies that protonation changes the symmetry of the molecular structure, and thus affects the interfacial potential drops. Fig. 5b shows a comparison of the calculated energy offsets of

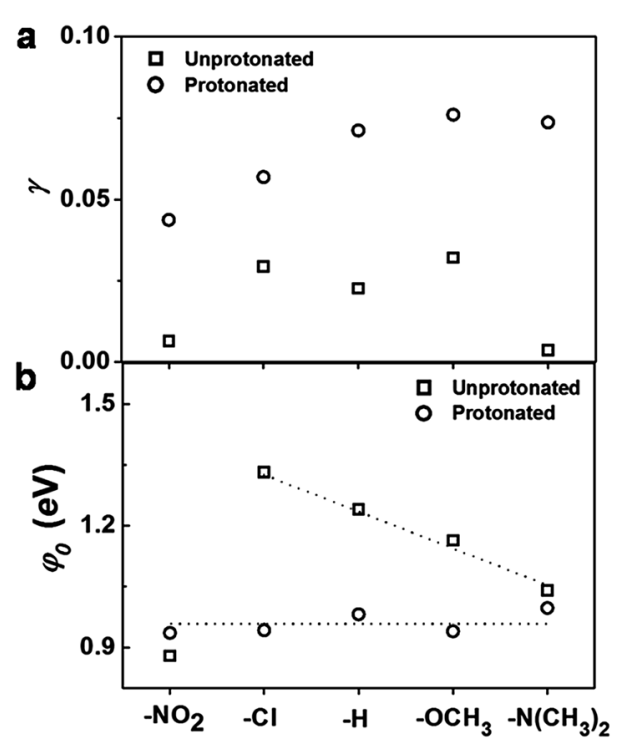

Fig. 5 Transition voltage analysis. The calculated voltage division factor $(\gamma)(a)$ and energy offset $\left(\varphi_{0}\right)(b)$ of unprotonated and protonated PPC- $\mathrm{NO}_{2}, \mathrm{PPC}-\mathrm{Cl}, \mathrm{PPC}-\mathrm{H}, \mathrm{PPC}-\mathrm{OCH}_{3}$ and PPC-N $\left(\mathrm{CH}_{3}\right)_{2}$ molecular junctions. the molecular wires before and after protonation. We can see that protonation converts the $\varphi_{0}$ value of different molecules to the same value within the experimental uncertainty. This is consistent with the observed trend of conductance value, demonstrating that the protonation indeed functions as a switch by changing the energy levels. Our previous study ${ }^{\mathbf{1 7}}$ showed that the tunneling barrier of the unprotonated PPC-molecular wires followed the order: $\varphi_{0}(-\mathrm{Cl})>\varphi_{0}(-\mathrm{H})>$ $\varphi_{0}\left(-\mathrm{OCH}_{3}\right)>\varphi_{0}\left(\mathrm{~N}\left(-\mathrm{CH}_{3}\right)_{2}\right)$, which agreed well with the observed order of conductance: $G(-\mathrm{Cl})<G(-\mathrm{H})<G\left(-\mathrm{OCH}_{3}\right)<G\left(\mathrm{~N}\left(-\mathrm{CH}_{3}\right)_{2}\right)$. This was attributed to the modulation of the charge tunneling barriers of the pentaphenylene wire via the pyridine unit, acting like a gate electrode. Since the pentaphenylene unit is a p-type semiconductor, the measured $\varphi_{0}$ reflects the $E_{\mathrm{F}}-E_{\text {Hомо }}$ offset. However, for the unprotonated PPC- $\mathrm{NO}_{2}$, its conductance was observed to be the lowest among the neutral compounds while its tunneling barrier was also the lowest. We attributed the cause of this phenomenon to a switch of transport mechanism by tunneling through the LUMO due to the strong electron withdrawing ability of the nitro group. The DFT calculations supported this explanation. ${ }^{17}$ The results in this study indicate that the effect of protonation is the same as for the nitro groups. The positive charge in the pyridine ring converted the molecular wires into n-type semiconductors. The charges are tunneled through the LUMO energy level. This mechanistic change in charge tunneling is the origin of the switching behavior.

The protonation effect observed in this work is different from those molecules in which the pyridine ring is a component along the charge transport pathway in a molecular wire. ${ }^{2,4}$ In this system, the pyridine ring is orthogonal to the conjugated system in a molecular wire. The effect of protonation is akin to a gating voltage, demonstrating that the molecular system reported here will be a genuine transistor if it can be connected to three electrodes.

\section{Conclusions}

The influence of protonation on the edge-on gating effect of a series of novel pyridinocyclophane moiety-based molecular junctions was investigated. It was found that the protonation on the nitrogen atom of the edge-on pyridine ring can reversibly change the conductance of the molecular wires and act as a switch. Protonation converted a molecular wire from a p-type to an n-type semiconductor. The switch mechanism is thus attributed to the change in charge tunneling channels from HOMO in neutral molecules to LUMO in protonated ones.

\section{Acknowledgements}

This work was mainly supported by NSF (DMR-1505130) and partially by NSF (DMR-1263006) and AFSOR. This work also benefited from NSF MRSEC at the University of Chicago.

\section{Notes and references}

1 P. Jiang, G. M. Morales, W. You and L. Yu, Angew. Chem., Int. Ed., 2004, 43, 4471. 
2 G. M. Morales, P. Jiang, S. W. Yuan, Y. G. Lee, A. Sanchez, W. You and L. Yu, J. Am. Chem. Soc., 2005, 127, 10456.

3 M.-K. Ng, D.-C. Lee and L. Yu, J. Am. Chem. Soc., 2002, 124, 11862.

4 X. Xiao, B. Xu and N. Tao, J. Am. Chem. Soc., 2004, 126, 5370.

5 D. Roldan, V. Kaliginedi, S. Cobo, V. Kolivoska, C. Bucher, W. Hong, G. Royal and T. Wandlowski, J. Am. Chem. Soc., 2013, 135, 5974.

6 S. Y. Quek, M. Kamenetska, M. L. Steigerwald, H. J. Choi, S. G. Louie, M. S. Hybertsen, J. B. Neaton and L. Venkataraman, Nat. Nanotechnol., 2009, 4, 230.

7 X. Zhao, C. Huang, M. Gulcur, A. S. Batsanov, M. Baghernejad, W. Hong, M. R. Bryce and T. Wandlowski, Chem. Mater., 2013, 25, 4340.

8 M.-H. Jo, J. E. Grose, K. Baheti, M. M. Deshmukh, J. J. Sokol, E. M. Rumberger, D. N. Hendrickson, J. R. Long, H. Park and D. C. Ralph, Nano Lett., 2006, 6, 2014.

9 S. J. Tans, A. R. M. Verschueren and C. Dekker, Nature, 1998, 393, 49.

10 M. Di Ventra, S. T. Pantelides and N. D. Lang, Appl. Phys. Lett., 2000, 76, 3448.

11 Y. Cui and C. M. Lieber, Science, 2001, 291, 851.

12 L. Y. Jiao, L. Zhang, X. R. Wang, G. Diankov and H. J. Dai, Nature, 2009, 458, 877.

13 I. Díez-Pérez, J. Hihath, Y. Lee, L. Yu, L. Adamska, M. A. Kozhushner, I. I. Oleynik and N. Tao, Nat. Chem., 2009, 1, 635.

14 Z. Ioffe, T. Shamai, A. Ophir, G. Noy, I. Yutsis, K. Kfir, O. Cheshnovsky and Y. Selzer, Nat. Nanotechnol., 2008, 3, 727.

15 M. L. Perrin, F. Prins, C. A. Martin, A. J. Shaikh, R. Eelkema, J. H. van Esch, T. Briza, R. Kaplanek, V. Kral, J. M. van Ruitenbeek, H. S. J. van der Zant and D. Dulić, Angew. Chem., Int. Ed., 2011, 50, 11223.
16 J. R. Widawsky, W. Chen, H. Vázquez, T. Kim, R. Breslow, M. S. Hybertsen and L. Venkataraman, Nano Lett., 2013, 13, 2889.

17 W.-Y. Lo, W. Bi, L. Li, I. H. Jung and L. Yu, Nano Lett., 2015, 15, 958.

18 V. Boekelheide, K. Galuszko and K. S. Szeto, J. Am. Chem. Soc., 1974, 96, 1578.

19 V. Boekelheide and C. H. Tsai, Tetrahedron, 1976, 32, 423.

20 G. M. Barrow, J. Am. Chem. Soc., 1956, 78, 5802.

21 N. S. Golubev, S. N. Smirnov, V. A. Gindin, G. S. Denisov, H. Benedict and H.-H. Limbach, J. Am. Chem. Soc., 1994, 116, 12055.

22 B. Xu and N. J. Tao, Science, 2003, 301, 1221.

23 S. Y. Guo, J. Hihath, I. Diez-Perez and N. J. Tao, J. Am. Chem. Soc., 2011, 133, 19189.

24 J. M. Beebe, B. Kim, J. W. Gadzuk, C. D. Frisbie and J. G. Kushmerick, Phys. Rev. Lett., 2006, 97, 026801.

25 E. H. Huisman, C. M. Guédon, B. J. van Wees and S. J. van der Molen, Nano Lett., 2009, 9, 3909.

26 T. E. Stern, B. S. Gossling and R. H. Fowler, Proc. R. Soc. London, Ser. A, 1929, 124, 699.

27 J. M. Beebe, B. Kim, C. D. Frisbie and J. G. Kushmerick, ACS Nano, 2008, 2, 827.

28 B. Capozzi, J. Xia, O. Adak, E. J. Dell, Z.-F. Liu, J. C. Taylor, J. B. Neaton, L. M. Campos and L. Venkataraman, Nat. Nanotechnol., 2015, 10, 522.

29 I. Bâldea, Chem. Phys., 2012, 400, 65.

30 I. Bâldea, Phys. Rev. B: Condens. Matter Mater. Phys., 2012, 85, 035442.

31 M. Araidai and M. Tsukada, Phys. Rev. B: Condens. Matter Mater. Phys., 2010, 81, 235114.

32 A. Vilan, D. Cahen and E. Kraisler, ACS Nano, 2013, 7, 695.

33 Z. Xie, I. Bâldea, C. E. Smith and C. D. Frisbie, ACS Nano, 2015, 9, 8022.

34 L. Xiang, T. Hines, J. L. Palma, X. Lu, V. Mujica, M. A. Ratner, G. Zhou and N. Tao, J. Am. Chem. Soc., 2016, 138, 679. 